\title{
Selective Decay and Coherent Vortices in Two-Dimensional Incompressible Turbulence
}

\author{
William H. Matthaeus, W. Troy Stribling, Daniel Martinez, and Sean Oughton \\ Bartol Research Institute, University of Delaware, Newark, Delaware 19716 \\ David Montgomery \\ Department of Physics and Astronomy, Dartmouth College, Hanover, New Hampshire 03755
}

(Received 14 May 1990)

\begin{abstract}
Numerical solution of two-dimensional incompressible hydrodynamics shows that states of near minimal ratio of enstrophy to energy can be attained in times short compared with the flow decay time, confirming the simplest turbulent selective decay conjecture, and suggesting that coherent vortex structures do not terminate nonlinear processes. After all possible vortex mergers occur, the vorticity attains a particlelike character, suggested by the late-time similarity of the streamlines to Ewald potential contours.

PACS numbers: $47.25 .-\mathrm{c}$
\end{abstract}

Mechanisms for turbulent relaxation known as selective decay processes ${ }^{1-5}$ have been proposed as a dynamical explanation for relaxation of toroidal plasma discharges and as a basic property of decaying turbulence in magnetohydrodynamics (MHD) and in two-dimensional (2D) hydrodynamics. A common feature of these conjectures is the prediction that the flows evolve towards a state in which ratios of certain ideal quadratic global invariants attain extremal values. In the simplest case of 2D hydrodynamics, selective decay implies evolution to a state of minimal $\Omega / E$ (enstrophy/energy) and the theory is closely related to the dynamical evolution suggested by Batchelor. ${ }^{6}$ At the present time, even though rigorous proofs of their validity are unavailable, selective decay theories stand as useful hypotheses that have been supported by a number of computational studies in a variety of systems. ${ }^{2,4,5,7}$ As far as we are aware, however, there have been no reported simulations that have followed decaying turbulence for sufficiently long times, at high enough Reynolds number $(R)$, and at high enough spatial resolution to conclude unambiguously that the flow attains the conjectured final state due to turbulence processes, rather than because of the persistence of the largest eddies. Moreover, recently, the suggestion has been made ${ }^{8,9}$ that, at high $R$, the emergence of isolated, possibly stable, ${ }^{10}$ coherent structures, i.e., isolated vortices, thwarts the selective decay process in 2D hydrodynamics by prematurely and permanently eliminating the nonlinear couplings that give rise to turbulence. This raises crucial questions regarding the applicability of turbulent relaxation mechanisms to 2D flows and to a variety of other high-Reynolds number, nonlinear, fluid systems. ${ }^{11}$

In this Letter we investigate the long-time behavior of the selective decay process, and its relationship to the effects of coherent vortex structures, by direct spectral method solutions of the 2D Navier-Stokes equations. We emphasize the quasiuniversal character of the final nonuniform state, which may be achieved as a consequence of a variety of different turbulent evolutionary scenarios. We show that turbulent selective decay proceeds due to progressive stretching and merger of vortex structures. By 220 initial eddy turnover times, all possible vortex mergers occur, and the flow closely approaches the final state predicted by selective decay, ${ }^{2}$ a state largely consistent with Batchelor's ${ }^{6}$ earlier theory for the unbounded case. There remains an unsettled issue pertaining to the degree of point vortexlike concentration remaining at late times. However, the attainment of this state contradicts McWilliams's conjecture ${ }^{8}$ that coherent structures "arrest" turbulence at much earlier times.

For 2D hydrodynamics at large $R$, the selective decay scenario involves acceleration of the decay rate of enstrophy (mean-square vorticity) while kinetic energy is approximately conserved. This leads to an energy spectrum progressively peaked at the longest wavelength, and, with specified boundary conditions, a clear prediction for the long-time flow conditions, which are predicted to emerge long before the period of final linear viscous decay. In a finite-size periodic box, the energy is predicted to concentrate into two counter-rotating vortices of the longest allowed wavelength. In this simplest instance of selective decay, energy admits an affinity for nonlinear back transfer in wave number, while enstrophy preferentially transfers to short wavelengths. Similar energy condensation effects are seen in the ideal equilibrium statistical mechanics of 2D flow, and in the inverse energy cascade of steady driven 2D flows. ${ }^{11,12}$

To investigate the time $(t)$ evolution of 2D hydrodynamic turbulence, we adopt a $2 \pi$-periodic geometry in the $(x, y)$ plane, with the fluid velocity $\mathbf{v}(x, y, t)$, and all other variables independent of the normal $\hat{\mathbf{z}}=\hat{\mathbf{x}} \times \hat{\mathbf{y}}$ coordinate. Numerical solutions are obtained by solving the equation for the scalar vorticity $\omega=(\nabla \times \mathbf{v})_{z}$,

$$
\frac{\partial \omega}{\partial t}+\mathbf{v} \cdot \nabla \omega=v \nabla^{2} \omega
$$

using a Fourier-Galerkin method of the Orszag-Patterson type. ${ }^{13}$ The kinematic viscosity $(v)$, in these dimensionless units, acts as the reciprocal of a nominal large- 
scale Reynolds number $1 / R$, using unit length and unit initial characteristic velocity. The method is based on a Fourier decomposition $\omega(\mathbf{x}, t)=\sum_{k} \omega(\mathbf{k}, t) \exp (i \mathbf{k} \cdot \mathbf{x})$ with integer components of $\mathbf{k}$. The results described below utilize a fully dealiased ${ }^{13} 512^{2}$ code with maximum retained wave number $\approx 241$ and minimum wave number $k_{\min } \equiv 1$. The modal energy spectrum $E(\mathbf{k})$ $\equiv|\mathbf{v}(\mathbf{k})|^{2} / 2$ is initialized according to $E(\mathbf{k})=C /[1+(k /$ $6)^{4}$ ], for $1 \leq k \leq 120$ (zero otherwise) using a Gaussian random number generator, corresponding to an omnidirectional energy spectrum $2 \pi k E(\mathbf{k})$ that falls off as $k^{-3}$. The constant $C$ is chosen to make the total energy per unit mass $E=\left\langle v^{2} / 2\right\rangle=\frac{1}{2} \sum_{\mathbf{k}} k^{-2}|\omega(\mathbf{k})|^{2}$ have the initial value $\frac{1}{2}(\langle\cdots\rangle$ denotes a volume average). The enstrophy $\Omega \equiv\left\langle\omega^{2}\right\rangle / 2=\frac{1}{2} \sum_{\mathbf{k}}|\omega(\mathbf{k})|^{2}$ has an initial value of 67. The palinstrophy $P \equiv \frac{1}{2} \sum_{\mathrm{k}} k^{2}|\omega(\mathbf{k})|^{2}$ is initially $=1.61 \times 10^{5}$. This closely emulates the initial spectrum used by McWilliams. ${ }^{8}$

Here we report on the run of longest duration and highest Reynolds number and spatial resolution we have carried out, with $R=14286$, corresponding to an initial microscale (enstrophy cascade) Reynolds number ${ }^{14} R_{\lambda}$ $\equiv \Omega^{3 / 2} / 2 v P \approx 24.5$. The computation extends beyond time $t=236$ (using an explicit method with $\Delta t=\frac{1}{2048}$, and no smoothing or hyperviscosity), using the $512^{2}$. resolution code and initial data described above. Shorter, smaller, and lower- $R$ runs showed extremely similar behavior to the extent that comparisons were possible.

The most direct consequence of selective decay, ${ }^{2}$ the rapid decrease of $\Omega$ relative to $E$, is clearly seen throughout the computation. For example, by $t=230, \Omega$ has decreased to 1.08 ( $1.6 \%$ of its initial value), whereas $E$ has decayed to 0.42 ( $84 \%$ of its initial value). However, since $\dot{E}=-2 v \Omega$ and $\dot{\Omega}=-2 v P$, one can easily see that $d(\Omega / E) / d t \leq 0$ for all $2 \mathrm{D}$ flows, ${ }^{5}$ whether turbulent or not. Thus, decrease of $\Omega / E$ is not alone sufficient to conclude that turbulent dynamics are important. However, the monotonic decay of $\Omega / E$ does imply that the dynamics are not frozen out into any single-wavenumber state with $\Omega / E=k_{1}^{2}>k_{\min }^{2}$ during the run, as might be expected from the discussion in Ref. 8 .

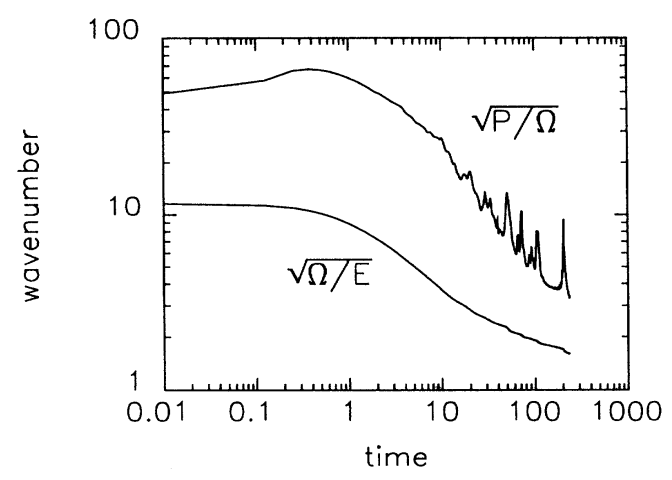

FIG. 1. Time evolution of $\sqrt{P / \Omega}$ and $\sqrt{\Omega / E}$.
A more complete picture can be seen from comparison of the behavior of the mean wave numbers $\sqrt{P / \Omega}$ and $\sqrt{\Omega / E}$, shown in Fig. 1. Apart from a factor involving the viscosity, the squares of the ratios in Fig. 1 can be interpreted as the decay rates (logarithmic time derivative) of $\Omega$ and $E$, respectively. ${ }^{2}$ For a dynamically frozen single-wave-number state, the ratio of these decay rates would be unity, and for linear decay of any spectral distribution, the same ratio would necessarily approach unity. Thus, Fig. 1 implies that the characteristic time for decay of enstrophy remains much shorter than that of the energy throughout the simulation. To maintain this condition, nonlinear interactions must persist in supporting the value of $P$ against rapid decay, by transfer of excitations to high wave number.

Characteristic wave-number spectra in selective decay indicate both direct transfer to high $k$ and back transfer of energy to the longest allowed wavelength $2 \pi / k_{\mathrm{min}}$. A sequence of modal spectra of energy and enstrophy in Fig. 2 illustrate these features. The spectra remain broadband, though steepened at high $k$. The energy in $k_{\text {min }}$ modes $[E(k=1)]$ increases steadily (but not monotonically) throughout the computed time, with $E(k=1)$ $=0.005$ at $t=1$, representing about $1 \%$ of the total $E$, while at $t=236, E(k=1)=0.307$, which is $\approx 74 \%$ of the total energy. The peak in the enstrophy spectrum at $k>1$, clearly seen in Fig. 2(a) at $t=1$, disappears by about $t=9$, and by $t=72$ [Fig. 2(b)] the enstrophy spectrum has a bimodal appearance, with the major peak at $k=1$ representing an energy-back-transfer subregion. A steepened direct-transfer region appears in the same figure at $k>10$. At later times [e.g., $t=236$ in Fig. 2 (c)] the domination of the spectral features at low $k$ is even more pronounced. Given the enhancement of $k_{\text {min }}$ excitations, these features are not explained by viscous decay.

The sequence of events in configuration $(x)$ space is described below in a time sequence of contour plots of vorticity (Fig. 3) and of stream function $\psi$ (Fig. 4; $\mathbf{v}=\boldsymbol{\nabla} \times \hat{\mathbf{z}} \psi)$. Prior to about $t=20$ [Fig. 3(a)] the vorticity dynamics are characterized by sheetlike structures

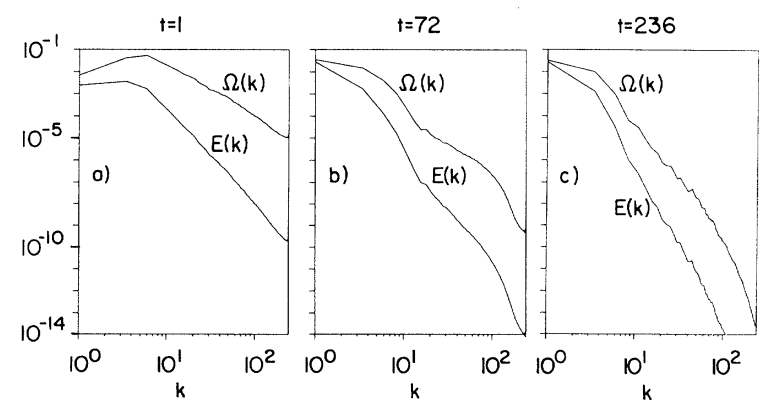

FIG. 2. Modal spectra of energy $E$ and enstrophy $\Omega$ at simulation times (a) $t=1$, (b) $t=72$, and (c) $t=236$, sorted into unit-width wave-number bins. 


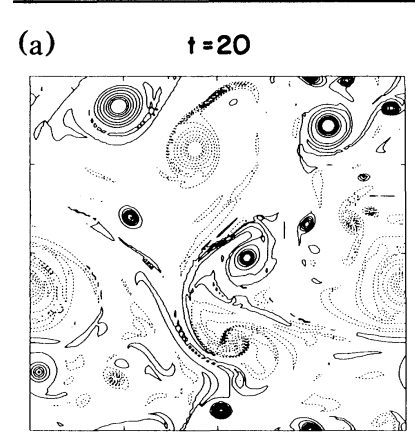

(c) $t=104$

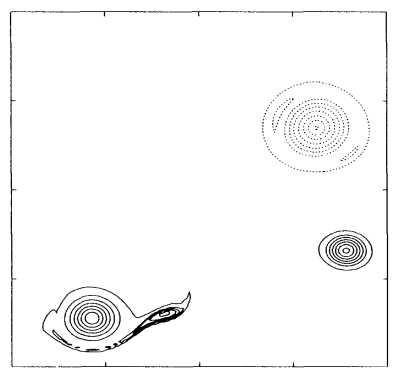

(b)

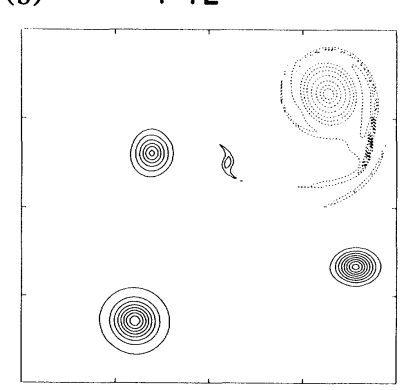

(d)

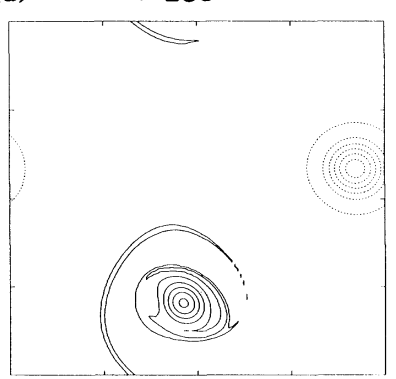

FIG. 3. Equally spaced contours of $\omega$ at (a) $t=20$, (b) $t=72$, (c) $t=104$, and (d) $t=208$. Data are averaged onto a $128^{2}$ grid for plotting purposes only.

that continually roll up around emerging centers of vorticity concentration, as has been described previously. ${ }^{8,9,14}$ The vorticity centers also "collide" and merge into larger structures rapidly at early times. After about $t=30$ the number of vorticity concentrations has been significantly reduced, and the amplitude of the sheetlike structures linking them has also decreased. Thus, one may describe, in a qualitative way, the situation as coming to resemble a system of emerging isolated vortices. The early-time dynamics of the vorticity has been discussed in detail by Brachet et al., ${ }^{9}$ and the emergence of the "isolated" vortices has been described in a similar computation by McWilliams. ${ }^{8}$ The conclusion of the latter work was that the emergence of isolated vortices acts to terminate the turbulent phase of 2D turbulence, thus halting relaxation processes, i.e., selective decay, that depend on continuation of strong nonlinear couplings.

The present computation, which is carried out to significantly longer times than those in either Ref. 8 or 9, shows that the coherent vortices have an important influence on the dynamics, but they do not halt the selective decay process. To achieve the final selective decay state, the vortices must continue to merge due to turbulence, driving the flow towards a nonturbulent state dominated by excitations at $k_{\text {min. }}$. The contour plots show that this indeed occurs. By about $t=72$ [Fig. $3(\mathrm{~b})$ ], all the major (nonsheetlike) negative-vortex concentrations have merged into a single strong coherent vortex. At the same time, there remain three strong positive vortices. The stream-function plots [Fig. 4(a)] indi-

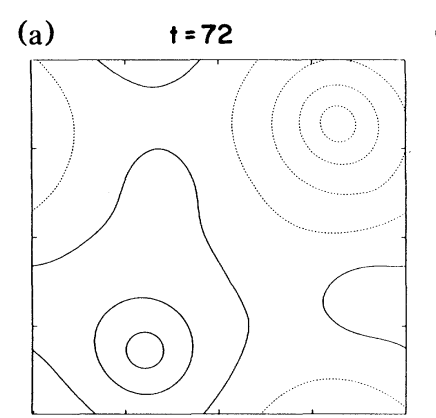

(c)
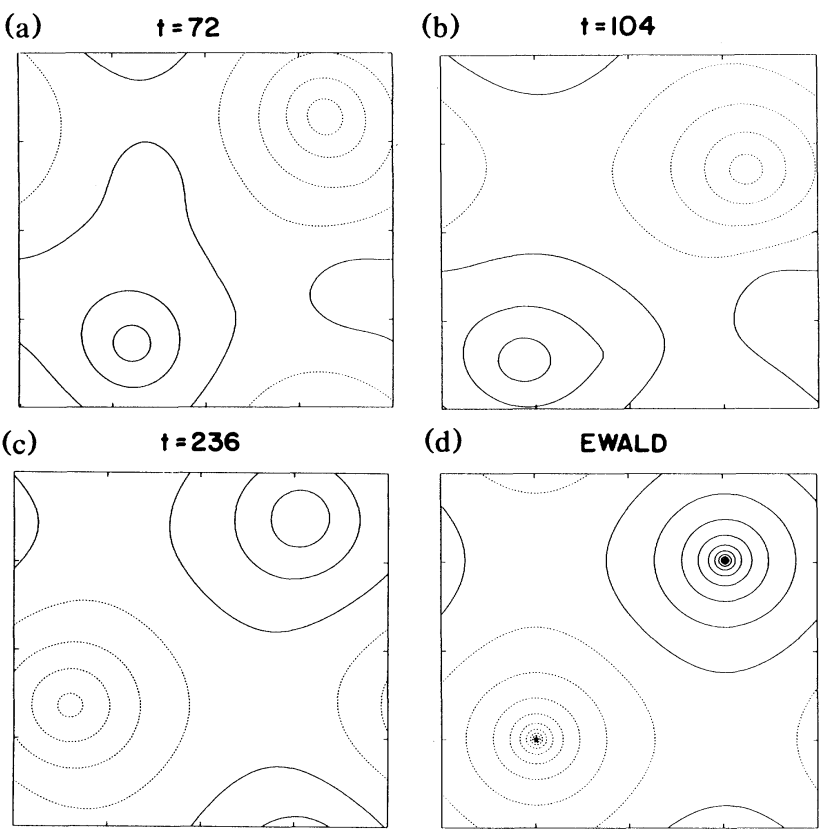

(d)

EWALD

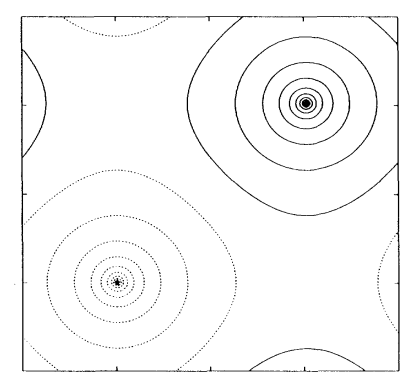

FIG. 4. Contour plots of $\psi$ at (a) $t=72$, (b) $t=104$, and (c) $t=236$. Panel (d) shows equipotentials of unit charges (vortices) placed at the equilibrium positions of a $2 \mathrm{D}$ Ewald potential.

cate that the flow has already become dominated by two large, though distorted, flow patterns. Two of the remaining positive-vorticity structures undergo merger in a "collision" that occurs just prior to $t=104$, clearly evidenced in the vorticity [Fig. 3(c)] and giving rise to an even more regular stream-function pattern [Fig. 4(b)]. Each of these identifiable mergers is associated with a significant transient increase in the palinstrophy, the influence of which is clearly seen in Fig. 1. Subsequently, the last two positive vortices merge at $t \approx 204$. The late stage of this interaction is shown in Fig. 3(d). Once again, there is a significant increase in $P$ during the merger. This is the last possible event of this type that can occur. The two remaining vortices, one positive and one negative, settle down to nearly circular cross sections thereafter, with their spatial separation being very nearly maximum along the diagonal of the periodic box. The highly regular contours of the stream function after the last merger [shown in Fig. 4(c)] show two large counter-rotating flow structures, visually almost indistinguishable from a pattern consisting entirely of $k_{\min }$ excitations.

Given that all possible mergers of coherent vortices are accomplished, and that the $\Omega / E$ ratio is reduced to a value of 2.57 (the minimum possible value $=1$ ), the flow, by $t=230$, has achieved most of what is expected in the selective decay picture of the dynamics. It seems implausible, even though there remains some excitation in 
$k>k_{\min }$, that the two remaining vorticity structures can ever break apart after this time. This viewpoint is made more clear by recalling the analogy between fully concentrated 2D discrete vortices ${ }^{15}$ and electrostatic guiding-center line charges, ${ }^{16}$ since the opposite-signed charges will effectively repel each other, at high energies, attempting to achieve an equilibrium with maximum possible separation. Taking periodicity into account, such an equilibrium is described by a latticelike configuration with the vortices located at the minima of each other's 2D Ewald potential, ${ }^{17}$ the charge separation at equilibrium being $\sqrt{2} \pi$, half the length of the box diagonal. In Fig. 4(d), potential contours are shown of line charges placed in the equilibrium Ewald lattice configuration. The similarity to the last $\psi$ contour plot at $t=236$ of the simulation is evident and is quite reasonable in view of the high degree of spatial concentration of the vorticity. At $t=236,81 \%$ of the negative vorticity (69.5\% of the positive vorticity) lies within a region of radius $0.72(=62$ simulation cells) of the position of the vorticity minimum (maximum), even though this region represents only $4.6 \%$ of the area of the box. Within the same regions lies $98.1 \%$ of the total enstrophy. Thus, one is left with somewhat of a paradoxical situation: Although the flow has very nearly approached the selective decay state, in terms of reduction of $\Omega / E$, the appearance of large flow patterns, and the merger all possible vortices, the surviving two vortex structures have retained, to some degree, a pointlike nature. They are, of course, not strictly point vortices, but are concentrated in space to very nearly the maximum extent consistent with the computed values of $E$ and $\Omega$. This Ewald lattice configuration is suggestive of a negative-temperature state of the discrete line-vortex model. ${ }^{15-17}$ Presumably, the subsequent dynamics after $t=236$ is relatively uneventful, and consists largely of small oscillations about this quasiequilibrium, and slow decay towards a $k_{\text {min }}$ state on a viscous decay time scale $\sim 1 / v k_{\text {min }}^{2}$ $\approx 1.4 \times 10^{4}$. It remains a challenge for theory to describe how this pointlike, spatially coherent, character of the vorticity can be reconciled with the observed, apparently inevitable, evolution towards the selective decayed state. Potentially large differences between decaying and driven spectra are suggested, to the extent that random driving may destroy coherence of the vortices.

The simulation described above presents a firm counterexample to the proposition ${ }^{8,9}$ that coherent vorticity structures prevent evolution towards an extremal selectively decayed state in a time much shorter than the viscous flow lifetime. The persistence of turbulent dynamics in attaining the final state reported here is unanticipated in previous simulations ${ }^{8,9}$ that computed for much shorter times. However, it is also clear that the spatial coherence of the vorticity plays an important role in the nature and rate of evolution of the turbulence. Merger of coherent vortices causes bursts of nonlinear activity, evidenced by transient increases of $P$ (cf. Fig.
1). This lends a temporal intermittency to the turbulence. In addition, the overall rate of turbulent decay of enstrophy (not shown), after $t=10$ but prior to the last merger, appears to be reasonably close to a time dependence of $P \sim t^{-2}, \Omega \sim t^{-1}$. In contrast, the previously unexamined (e.g., Ref. 9) similarity law of Batchelor ${ }^{6}$ predicts that $P \sim t^{-3}$ and $\Omega \sim t^{-2}$. Evidently, the spatially coherent, temporally intermittent nature of the vorticity interactions slows the turbulent evolution, in a way unforeseen in the Batchelor decay rates (Ref. 18 cites early-time slowing of turbulence due to coherent vortices). A full treatment of the statistics of 2D turbulent decay needs to take into account the influence of both selective decay and coherent vortices in a way ${ }^{16}$ that goes beyond simple inequalities in global decay rates.

This research has been supported in part by NSF Grant No. ATM-89131627 at Bartol, and NASA Grant No. NAG-W-710 and DOE Grants No. DEFG$0285 E R 53194$ and No. DEFG0285ER53298 at Dartmouth. Computations were supported by the NSF San Diego Supercomputing Center.

'D. Montgomery, L. Turner, and G. Vahala, Phys. Fluids 21, 757 (1978).

${ }^{2}$ W. Matthaeus and D. Montgomery, Annu. N.Y. Acad. Sci. 357, 203 (1980).

${ }^{3}$ W. Matthaeus and D. Montgomery, Statistical Physics and Chaos in Fusion Plasmas, edited by C. Horton and L. Reichl (Wiley, New York, 1984), p. 285.

${ }^{4}$ S. Riyolpoulos, A. Bondeson, and D. Montgomery, Phys. Fluids 25, 757 (1982).

${ }^{5}$ A. C. Ting, W. H. Matthaeus, and D. Montgomery, Phys. Fluids 29, 3261 (1986).

${ }^{6}$ G. K. Batchelor, Phys. Fluids, Suppl. II, 12, 233 (1969).

${ }^{7}$ J. Dahlburg, D. Montgomery, D. Doolen, and L. Turner, Phys. Rev. Lett. 57, 428 (1986); J. Plasma Phys. 37, 299 (1987).

${ }^{8}$ J. C. McWilliams, J. Fluid Mech. 146, 21 (1984).

${ }^{9}$ M. Brachet, M. Meneguzzi, H. Politano, and P.-L. Sulem, J. Fluid Mech. 194, 333 (1988).

${ }^{10}$ C. E. Leith, Phys. Fluids 27, 1388 (1984).

${ }^{11}$ R. H. Kraichnan, Phys. Fluids 10, 1417 (1967); R. H. Kraichnan and D. Montgomery, Rep. Prog. Phys. 43, 547 (1980); A. Hasegawa, Adv. Phys. 34, 1 (1985).

${ }^{12}$ D. K. Lilly, Phys. Fluids, Suppl. II, 12, 240 (1969); D. Fyfe, D. Montgomery, and G. Joyce, J. Plasma Phys. 17, 369 (1977); M. Hossain, W. Matthaeus, and D. Montgomery, J. Plasma Phys. 30, 479 (1983).

${ }^{13}$ S. Orszag, Stud. Appl. Math. 50, 243 (1971); G. Patterson and S. Orszag, Phys. Fluids 14, 2353 (1971).

${ }^{14}$ J. Herring, S. Orszag, R. Kraichnan, and D. Fox, J. Fluid Mech. 66, 417 (1974).

${ }^{15}$ L. Onsager, Nuovo Cimento Suppl. 6, 279 (1949).

${ }^{16} \mathrm{G}$. Joyce and D. Montgomery, J. Plasma Phys. 10, 107 (1973).

${ }^{17}$ C. E. Seyler, Phys. Fluids 19, 1336 (1976); Phys. Rev. Lett. 32, 515 (1974).

${ }^{18}$ J. C. McWilliams, Phys. Fluids A 2, 547 (1990). 